\title{
Aplicação do método Bayesiano na estimação de correlações genéticas e fenotípicas de peso em codornas de corte em várias idades ${ }^{1}$
}

\section{Eliane Maria Wilbert Winter ${ }^{2}$, Marina Isabel Mateus de Almeida ${ }^{3}$, Edson Gonçalves de Oliveira ${ }^{4}$, Elias Nunes Martins ${ }^{5}$, Andressa Santanna Natel ${ }^{6}$, Diego Surek ${ }^{6}$}

\author{
1 Parte da dissertação de Mestrado da primeira autora (Curso de pós-graduação em Genética, área de concentração em Melhoramento Animal, \\ Universidade Federal do Paraná). Trabalho financiado pelo CNPq. \\ 2 Mestranda do Departamento de Genética da Universidade Federal do Paraná \\ ${ }^{3}$ Departamento de Genética da Universidade Federal do Paraná - Orientadora. \\ ${ }^{4}$ Departamento de Zootecnia da Universidade Federal do Paraná. \\ ${ }^{5}$ Departamento de Zootecnia da Universidade Estadual de Maringá. \\ 6 Graduação do Departamento de Zootecnia da Universidade Federal do Paraná.
}

RESUMO - Objetivou-se com este estudo obter estimativas de correlações genéticas e fenotípicas para características peso aos 7 dias (PES7), peso aos 14 dias (PES14), peso aos 28 dias (PES28) e peso aos 42 dias de idade (PES42) de codornas de corte. No galpão de criação, foram alojadas 1.022 aves devidamente identificadas, utilizadas para avaliação do desempenho produtivo, por meio de pesagens individuais. Os componentes de (co)variância e os parâmetros genéticos foram estimados por procedimentos Bayesianos via amostrador de Gibbs utilizando-se o programa MTGSAM. As estimativas de correlações genéticas obtidas foram de 0,73 entre PES7 e PES14, 0,52 entre PES7 e PES28, 0,55 entre PES7 e PES42, 0,73 entre PES14 e PES28 e 0,56 entre PES14 e PES42 dias de idade. Correlação genética de 0,81 entre os pesos de 28 e 42 dias de idade indica a possibilidade de aumento do peso corporal aos 42 dias de idade pela seleção do peso corporal aos 28 dias de idade. As correlações fenotípicas variaram de 0,57 a 0,85 e foram ligeiramente superiores às genéticas, sugerindo que a correlação de ambiente é de pequena magnitude e positiva. Os menores intervalos de credibilidade das estimativas de correlações genéticas foram entre os pesos aos 28 e 42 dias e aos 14 e 28 dias.

Palavras-chave: amostrador de Gibbs, codornas de corte, correlações genéticas, peso

\section{Application of the Bayesian method to estimate genetic and phenotypic parameters of growth traits of meat quails}

\begin{abstract}
The objectives of this study were to estimate phenotypic and genetic parameters for growth traits of meat quails. A total of 1022 birds were housed, identified and individually weighted at 7 days (PES7); 14 days (PES14); 28 days (PES28) and at 42 days (PES42). Covariance components and genetic parameters were estimated by Bayesian procedures using Gibbs sampling with MTGSAM software. Genetic correlations between PES7 and PES14, PES28, and PES42 were respectively $0.73,0.52$ and 0.55 and between PES14 and PES28 and PES42 were respectively 0.73 and 0.56 . Genetic correlation of 0.81 between PES28 and PES42 days indicates the possibility to increase weight at 42 days by selecting for weight at 28 days of age. Estimates of phenotypic correlations ranged from 0.57 to 0.85 and were larger than the genetic ones suggesting small magnitude of positive environmental correlation estimates. The smallest credibility intervals were observed for genetic correlations between PES28 and PES42 and between PES14 and PES28.
\end{abstract}

Key Words: genetic correlations, Gibbs sampling, meat quails, weight

\section{Introdução}

O constante crescimento populacional vem estimulando a exploração de outras aves como novas opções de fontes protéicas. A demanda por carne de melhor qualidade de um mercado consumidor exigente justifica o crescimento expressivo da produção de carne de codorna.

No Brasil, as codornas foram inicialmente utilizadas para produção de ovos, de modo que a produção de carne foi, durante longo tempo, uma atividade secundária caracterizada pelo abate de machos não utilizados na reprodução e de fêmeas de descarte, após o ciclo produtivo. Em geral, as carcaças obtidas eram pequenas e a carne era dura, prejudicando a qualidade do produto. Contudo, com a introdução de uma variedade européia que atende os requisitos necessários à produção de carne, a exploração para corte começou a ser difundida. A superioridade da linhagem européia como produtora de carne (peso $70 \%$ superior 
em relação à linhagem japonesa na idade adulta) foi constatada por Almeida et al. (2002). Sua exploração vem crescendo no Brasil de forma considerável. Este sucesso se justifica pela qualidade de sua carne, que apresenta características sensoriais de grande aceitabilidade pelo consumidor (Oliveira et al., 2005).

No entanto, o sucesso econômico da produção de carne não depende apenas das características qualitativas de carcaça. Aspectos quantitativos, como rendimentos e índices produtivos, devem ser considerados. A conformação de carcaça precisa ser melhorada, pois estas aves apresentam baixo rendimento de cortes nobres, como peito e pernas (Almeida et al., 2002). De acordo com Oliveira et al. (2002), seus índices produtivos são ainda baixos e a espécie apresenta altos valores de consumo de ração e conversão alimentar.

Martins (2002) cita que não existe ainda no Brasil qualquer programa de melhoramento de codornas desenvolvido em bases técnicas. A prática corrente tem sido a reprodução do material disponível, que, pela deficiência de controle e falta de esquemas de seleção adequados, sofre problemas de redução pela consangüinidade, resultando em diminuição de postura, queda de fertilidade e aumento de mortalidade. Com a perspectiva de formação de linhagens específicas para corte, torna-se necessário o desenvolvimento desses programas para que o potencial zootécnico desta espécie seja otimizado, visando à demanda por um produto de qualidade e baixo custo de produção. A avaliação da estrutura das populações disponíveis, por meio de informações sobre parâmetros genéticos, é imprescindível para se definirem estratégias de seleção. A importância do estudo de correlações consiste em analisar a influência da seleção para determinada característica sobre outras características.

Com a evolução na área computacional e o desenvolvimento de novas teorias, ampliou-se a possibilidade de escolha dos procedimentos de estimação de componentes de (co)variância. O método de máxima verossimilhança restrita (REML) tornou-se uma opção bastante atrativa e tem sido amplamente empregado em melhoramento animal. Recentemente, métodos Bayesianos vêm sendo propostos como uma opção na avaliação do mérito genético de animais, possibilitando a obtenção de estimativas pontuais e intervalos de credibilidade para as distribuições a posteriori dos parâmetros, sem aproximações ou uso de pressuposições de normalidade, representando uma vantagem sobre os métodos clássicos, como o de máxima verossimilhança restrita (Falcão et al., 2004).

Os objetivos neste trabalho foram determinar as estimativas de correlações genéticas e fenotípicas pelo método
Bayesiano utilizando-se amostrador de Gibbs e construir intervalos de credibilidade e regiões de alta densidade para as estimativas de correlações genéticas para as características de desempenho de codornas de corte.

\section{Material e Métodos}

O experimento foi realizado nas instalações do Setor de Aves Alternativas da Fazenda Experimental Cangüiri da Universidade Federal do Paraná. Para formação da geração parental, foram adquiridos 670 pintos de codornas de corte (335 machos e 335 fêmeas) de origem européia com um dia de idade. Com a reprodução desta geração, obteve-se a primeira geração para formação do banco de dados.

Os pintos sexados foram alojados no galpão de criação e, durante sete dias, foram criados em círculos de proteção revestidos com cama de maravalha e providos com campânula a gás, propiciando temperatura de 32 a $35^{\circ} \mathrm{C}$. Aos sete dias de idade, as aves de cada sexo foram aleatoriamente distribuídas em duas baterias de quatro andares, totalizando oito gaiolas por bateria. Em cada gaiola, foram alojadas 38 codornas separadas por sexo. Até os 21 dias de idade, cada gaiola continha um sistema de aquecimento com lâmpada incandescente de 75 watts, propiciando temperatura de 32 a $35^{\circ} \mathrm{C}$. A alimentação foi fornecida à vontade e consistiu de uma ração única, farelada, com $24 \%$ de PB e $2.900 \mathrm{kcal}$ de EM, formulada com base nas recomendações do NRC (1994) para codornas em fase de crescimento.

Aos 35 dias de idade, 100 machos e 200 fêmeas, sorteados aleatoriamente, foram alojados no galpão de reprodução para se adaptarem ao novo ambiente. Em cada gaiola foram alojados um macho e duas fêmeas. Alguns dias antes da coleta dos ovos, as fêmeas foram separadas para possibilitar a identificação das mães. A coleta de ovos para incubação teve início na $34^{\mathrm{a}}$ semana e foi realizada por oito dias consecutivos, às $8 \mathrm{~h} 30$, totalizando 1.435 ovos. Na fase de coleta, os ovos foram devidamente identificados quanto à origem. Os ovos foram incubados por um período de 14 dias a temperatura de 37,3 a $37,5^{\circ} \mathrm{C}$ e umidade de $70 \%$.

Após este período, os ovos de cada casal foram colocados, de acordo com a identificação, em sacos de filó devidamente fechados para garantir a identidade de cada ave recém-eclodida. Esses sacos foram transferidos para o nascedouro, onde permaneceram durante três dias em temperatura de $37^{\circ} \mathrm{C}$ e umidade de $88 \%$. Decorridas as primeiras 24 horas da eclosão, as 1.179 aves anilhadas foram alojadas em 50 gaiolas no galpão de criação até aos 49 dias. A condução do experimento seguiu os mesmos procedimentos descritos neste trabalho para a geração dos pais. 
Neste trabalho, foram utilizados ao nascimento dois sistemas de numeração: colar feito com elástico de silicone no pescoço e etiqueta colante na perna. Ambos os sistemas apresentaram problemas: o colar causou desconforto nas aves, por sua entrada no bico, prejudicando a alimentação, e a etiqueta descolou com o calor. No quarto dia após o nascimento, optou-se pela remoção dos colares das aves que ainda não tinham perdido a etiqueta colante. Um novo anilhamento, feito com "arames de pão", foi realizado. Nesta fase de colocação da nova anilha, 57 codornas perderam sua identificação e foram excluídas do trabalho.

A avaliação do desempenho produtivo das aves foi feita por meio de pesagens individuais semanais. As características estudadas foram peso aos 7 (PES7), 14 (PES14), 28 (PES28) e 42 dias (PES42) de idade.

As estimativas das correlações genéticas e fenotípicas foram obtidas pelo método Bayesiano utilizando-se algoritmo amostrador de Gibbs, disponível no programa MTGSAM (Multiple Trait Gibbs Sampling in Animal Models), desenvolvido por Van Tassel \& Van Vleck (1996). Foi realizada análise multicaracter. Para análise do grupo de características de desempenho, foram incluídos no modelo o sexo dos animais como efeito fixo, o número de animais por gaiola como covariável e, como efeitos aleatórios, foram considerados o efeito genético aditivo e os efeitos aleatórios não-correlacionados de ambiente materno-permanente. As informações deste grupo foram analisadas com um arquivo de pedigree constituído de 1.312 codornas.

O modelo animal utilizado para análise foi:

$\left[\begin{array}{c}\mathrm{y}_{1} \\ \mathrm{y}_{2} \\ \mathrm{M} \\ \mathrm{y}_{\mathrm{i}}\end{array}\right]=\left[\begin{array}{cccc}\mathrm{X}_{1} & 0 & \Lambda & 0 \\ 0 & \mathrm{X}_{2} \Lambda & 0 \\ \mathrm{M} & \mathrm{M} & \mathrm{O} & \mathrm{M} \\ 0 & 0 & \Lambda & \mathrm{X}_{\mathrm{i}}\end{array}\right]\left[\begin{array}{c}\beta_{1} \\ \beta_{2} \\ \mathrm{M} \\ \beta_{\mathrm{i}}\end{array}\right]+\left[\begin{array}{cccc}\mathrm{Z}_{1} & 0 & \Lambda & 0 \\ 0 & \mathrm{Z}_{2} \Lambda & 0 \\ \mathrm{M} & \mathrm{M} & \mathrm{O} & \mathrm{M} \\ 0 & 0 & \Lambda & \mathrm{Z}_{\mathrm{i}}\end{array}\right]\left[\begin{array}{c}\mathrm{a}_{1} \\ \mathrm{a}_{2} \\ \mathrm{M} \\ \mathrm{a}_{\mathrm{i}}\end{array}\right]+\left[\begin{array}{cccc}\mathrm{W}_{1} & 0 & \Lambda & 0 \\ 0 & \mathrm{~W}_{2} \Lambda & 0 \\ \mathrm{M} & \mathrm{M} & \mathrm{O} & \mathrm{M} \\ 0 & 0 & \Lambda & \mathrm{W}_{\mathrm{i}}\end{array}\right]\left[\begin{array}{c}\mathrm{p}_{1} \\ \mathrm{p}_{2} \\ \mathrm{M} \\ \mathrm{p}_{\mathrm{i}}\end{array}\right]+\left[\begin{array}{c}\mathrm{e}_{1} \\ \mathrm{e}_{2} \\ \mathrm{M} \\ \mathrm{e}_{\mathrm{i}}\end{array}\right]$

em que $y_{1}, y_{2}, \ldots, y_{\mathrm{i}}=$ vetores de observações das i características; $\mathrm{X}_{1}, \mathrm{X}_{2}, \ldots, \mathrm{X}_{\mathrm{i}}=$ matrizes de incidência dos efeitos fixos nos vetores $y ; \beta_{1}, \beta_{2}, \ldots, \beta_{i}=$ vetores dos efeitos fixos; $Z_{1}, Z_{2}, \ldots, Z_{i}=$ matrizes de incidência dos valores genéticos nos vetores $y ; a_{1}, a_{2}, \ldots, a_{i}=$ vetores dos efeitos aleatórios dos valores genéticos; $\mathrm{W}_{1}, \mathrm{~W}_{2}, \ldots, \mathrm{W}_{\mathrm{i}}=$ matrizes de incidência dos efeitos de ambiente materno-permanente nos vetores $\mathrm{y} ; \mathrm{p}_{1}, \mathrm{p}_{2}, \ldots, \mathrm{p}_{\mathrm{i}}=$ vetores dos efeitos de ambiente maternopermanente; $\mathrm{e}_{1}, \mathrm{e}_{2}, \ldots, \mathrm{e}_{\mathrm{i}}=$ vetores dos erros aleatórios.

Cadeias de Gibbs de 7.000.000 de iterações foram geradas com descarte inicial de 1.000 .000 de iterações para o período de aquecimento da cadeia, no intuito de se minimizarem os efeitos dos valores iniciais. Para assegurar a independência da amostra, considerou-se um intervalo de retirada de 3.000 . A convergência das cadeias geradas pelo amostrador de Gibbs foi monitorada por meio de análise gráfica usando os testes de diagnóstico de Geweke, Raftery e Lewis e de Heidelberger e Welch, disponíveis no CODA (Convergence Diagnosis and Output Analysis) do programa R.

\section{Resultados e Discussão}

As estimativas das correlações fenotípicas obtidas entre as características analisadas apresentaram magnitude ligeiramente superior às genéticas (Tabela 1). Estes resultados estão de acordo com os reportados por Sefton \& Siegel (1974), nas correlações entre os pesos aos 7 com 42,14 com 28, 14 com 42 e 28 com 42 dias, e também com Aggrey \& Cheng (1994), nas correlações entre os pesos aos 7 com 14 e aos 7 com 28 dias, mas diferem das correlações fenotípicas inferiores às genéticas encontradas por Saatci et al. (2003). Embora neste trabalho não tenham sido estimadas as correlações de ambiente, a proximidade dos valores das correlações fenotípicas e genéticas sugere que a correlação de ambiente é de pequena magnitude e positiva.

A característica peso aos 7 dias apresentou alta correlação genética com peso aos 14 dias, dados consistentes com os descritos para fêmeas por Sefton \& Siegel (1974) e Saatci et al. (2003), que foi superior $(0,96)$, e por Aggrey \& Cheng (1994), que foi inferior $(0,64)$. Esta correlação indica que a seleção para maior peso aos 7 dias implica resposta no mesmo sentido no peso aos 14 dias. Entre os pesos aos 7 e aos 28 dias, a correlação genética foi moderada e de magnitude superior à estimativa de 0,45 encontrada por Aggrey \& Cheng (1994), enquanto as estimativas descritas para fêmeas por Sefton \& Siegel (1974) e Saatci et al. (2003) indicaram altas correlações genéticas $(0,82$ e 0,94 ,

Tabela 1 - Estimativas de correlações genéticas (acima da diagonal) e fenotípicas (abaixo da diagonal) entre as características analisadas

Table 1 - Estimates of genetic (above diagonal) and phenotypic correlations (below diagonal) among the analyzed traits

\begin{tabular}{lcccc}
\hline $\begin{array}{l}\text { Característica } \\
\text { Trait }\end{array}$ & Pes7 & Pes14 & Pes28 & Pes42 \\
\hline PES7 & - & 0,73 & 0,52 & 0,55 \\
PES14 & 0,81 & - & 0,73 & 0,56 \\
PES28 & 0,65 & 0,79 & - & 0,81 \\
PES42 & 0,57 & 0,66 & 0,85 & - \\
\hline
\end{tabular}

PES7 - peso aos sete dias; PES14 - peso aos 14 dias; PES28 - peso aos 28 dias; PES42 - peso aos 42 dias.

PES7 - weight at 7 days; PES14 - weight at 14 days; PES28 - weight at 28 days PES42 - weight at 42 days. 
respectivamente). O peso aos 7 dias também apresentou correlação genética moderada com peso aos 42 dias, diferindo da estimativa baixa $(0,33)$ obtida em fêmeas por Sefton \& Siegel $(1974)$ e da estimativa alta $(0,89)$ encontrada por Saatci et al. (2003).

Quanto ao peso aos 14 dias, o resultado de 0,73 indicou alta correlação genética com o peso aos 28 dias, semelhante às obtidas em fêmeas por Sefton \& Siegel (1974), Aggrey \& Cheng (1994) e Saatci et al. (2003), embora esses autores tenham obtido estimativas levemente superiores $(0,86$, 0,76 e 0,90 , respectivamente). Entre os pesos aos 14 e 42 dias, a correlação genética obtida foi de 0,56 , inferior ao valor de correlação genética de 0,73 encontrado por Saatci et al. (2003).

O peso aos 28 dias apresentou alta correlação genética $(0,81)$ com o peso aos 42 dias, o que está de acordo com os relatos de Sefton \& Siegel (1974), Brah et al. (1997) e Saatci et al. (2003). Por outro lado, estes resultados contrariam a baixa estimativa $(0,31)$ encontrada por Kumar et al. (2002). Estas correlações indicam que a seleção para peso aos 28 dias provoca resposta no mesmo sentido no peso aos 42 dias. As correlações genéticas obtidas entre os pesos em diferentes idades analisadas indicam que a seleção para peso pode ser realizada em idades anteriores. Embora em codornas exista pouca informação disponível, em linhagens de frangos de corte, a seleção precoce por várias gerações acarretou problemas metabólicos como, por exemplo, morte súbita e ascite como características negativamente correlacionadas (Burt, 2002).

As diferenças entre as estimativas de correlações genéticas e fenotípicas encontradas para a mesma característica nos diferentes estudos comparados podem ser atribuídas a fatores como diferenças entre as populações ou às condições sob as quais foram estudadas, diferenças entre os modelos de análise e a metodologia utilizada. Em nenhum dos trabalhos revisados para comparação das estimativas de correlações genéticas e fenotípicas, foi utilizada a metodologia Bayesiana.

$\mathrm{Na}$ Tabela 2 são apresentados os coeficientes de variação, os intervalos de credibilidade e as regiões de alta densidade a $90 \%$ para as correlações genéticas entre as características analisadas.

Os intervalos de credibilidade das estimativas de correlações genéticas entre os pesos aos 28 e 42 dias e aos 14 e 28 dias foram os que apresentaram as menores amplitudes de variação, o que pode ser confirmado pelos menores coeficientes de variação (7,40 e 14,09\%). Estes resultados permitem concluir que as estimativas destes parâmetros são mais precisas. Assim, por exemplo, para correlação genética entre peso aos 28 e 42 dias de idade, a probabilidade de que o valor da correlação genética esteja situado entre 0,70 e 0,88 é de $90 \%$. Se comparados os intervalos de credibilidade às regiões de alta densidade, nota-se que, para todas as estimativas de correlações genéticas das características analisadas, ocorre pequena assimetria, com predominância de valores altos do parâmetro. Estas observações sugerem que há maior probabilidade de os valores das correlações genéticas estarem mais próximos do limite superior que do limite inferior do intervalo. A variabilidade e assimetria são representadas na Figura 1, na qual se nota menor dispersão na correlação genética entre os pesos de 28 e 42 dias, seguida dos pesos de 14 e 28 dias, e maior dispersão entre os pesos de 7 e 28 dias. Observa-se assimetria à esquerda (mediana está mais próxima do terceiro quartil que do primeiro), indicando que esta distorção foi causada pela obtenção de alguns valores extremamente baixos.

A convergência das cadeias foi satisfeita, não existindo evidências contra a convergência pelos testes de diagnóstico de Geweke, Raftery e Lewis e de Heidelberger e Welch. Além dos testes de diagnóstico de convergência, a monitoração foi feita por meio de análise gráfica. Os valores das correlações genéticas ao longo das iterações (acima) e

Tabela 2 - Coeficiente de variação (\%), intervalo de credibilidade e região de alta densidade a 90\% para as estimativas de correlações genéticas das características analisadas

Table 2 - Coefficient of variation (\%), credibility interval and high density region at the level of $90 \%$ for genetic correlation estimates of analyzed traits

\begin{tabular}{lccc}
\hline $\begin{array}{l}\text { Característica correlacionada } \\
\text { Correlated trait }\end{array}$ & $\mathrm{CV}^{1}(\%)$ & $\begin{array}{c}\text { Intervalo de credibilidade } \\
\text { Credibility interval }\end{array}$ & $\begin{array}{c}\text { Região de alta densidade } \\
\text { Higth density region }\end{array}$ \\
\hline PES7 e PES14 & 18,83 & $0,46-0,87$ & $0,55-0,89$ \\
PES7 e PES28 & 38,02 & $0,11-0,76$ & $0,22-0,80$ \\
PES7 e PES42 & 32,68 & $0,20-0,77$ & $0,26-0,80$ \\
PES14 e PES28 & 14,09 & $0,52-0,85$ & $0,60-0,87$ \\
PES14 e PES42 & 24,51 & $0,30-0,72$ & $0,37-0,76$ \\
PES28 e PES42 & 7,40 & $0,70-0,88$ & $0,73-0,89$
\end{tabular}

PES7 - peso aos 7 dias; PES14 - peso aos 14 dias; PES28 - peso aos 28 dias; PES42 - peso aos 42 dias.

PES7 - weight at 7 days; PES14 - weight at 14 days; PES28 - weight at 28 days; PES42 - weight at 42 days.

${ }^{1}$ Coeficiente de variação.

1 Coefficient of variation. 


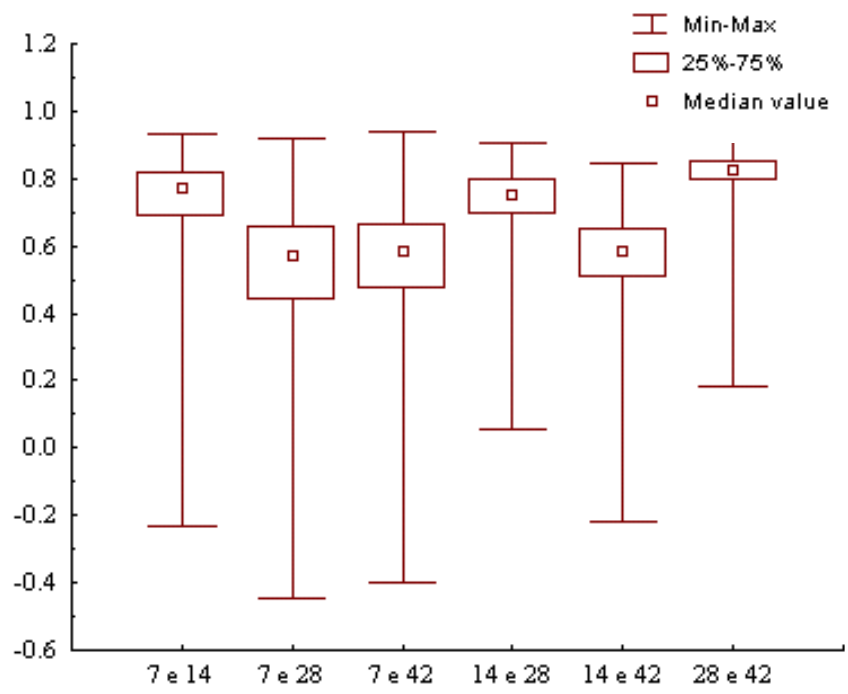

Figura 1 - Estimativas de correlações genéticas entre os pesos aos 7 e 14, aos 7 e 28, aos 7 e 42 , aos 14 e 28 , aos 14 e 42 e aos 28 e 42 dias de idade.

Figure 1 - Estimates of genetic correlation between the weights at 7 and 14, 7 and 28, 7 and 42, 14 and 28,14 and 42 and 28 and 42 days of age.

(a)
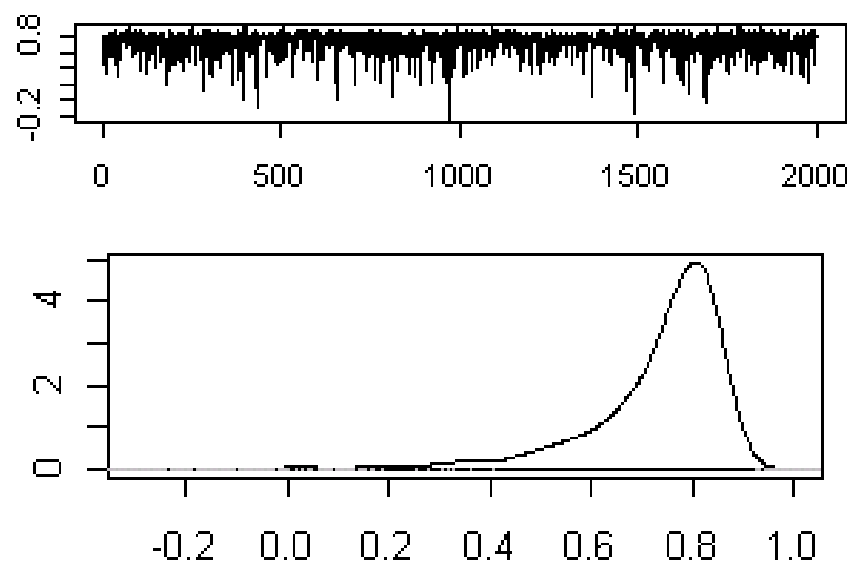

(c) (b)
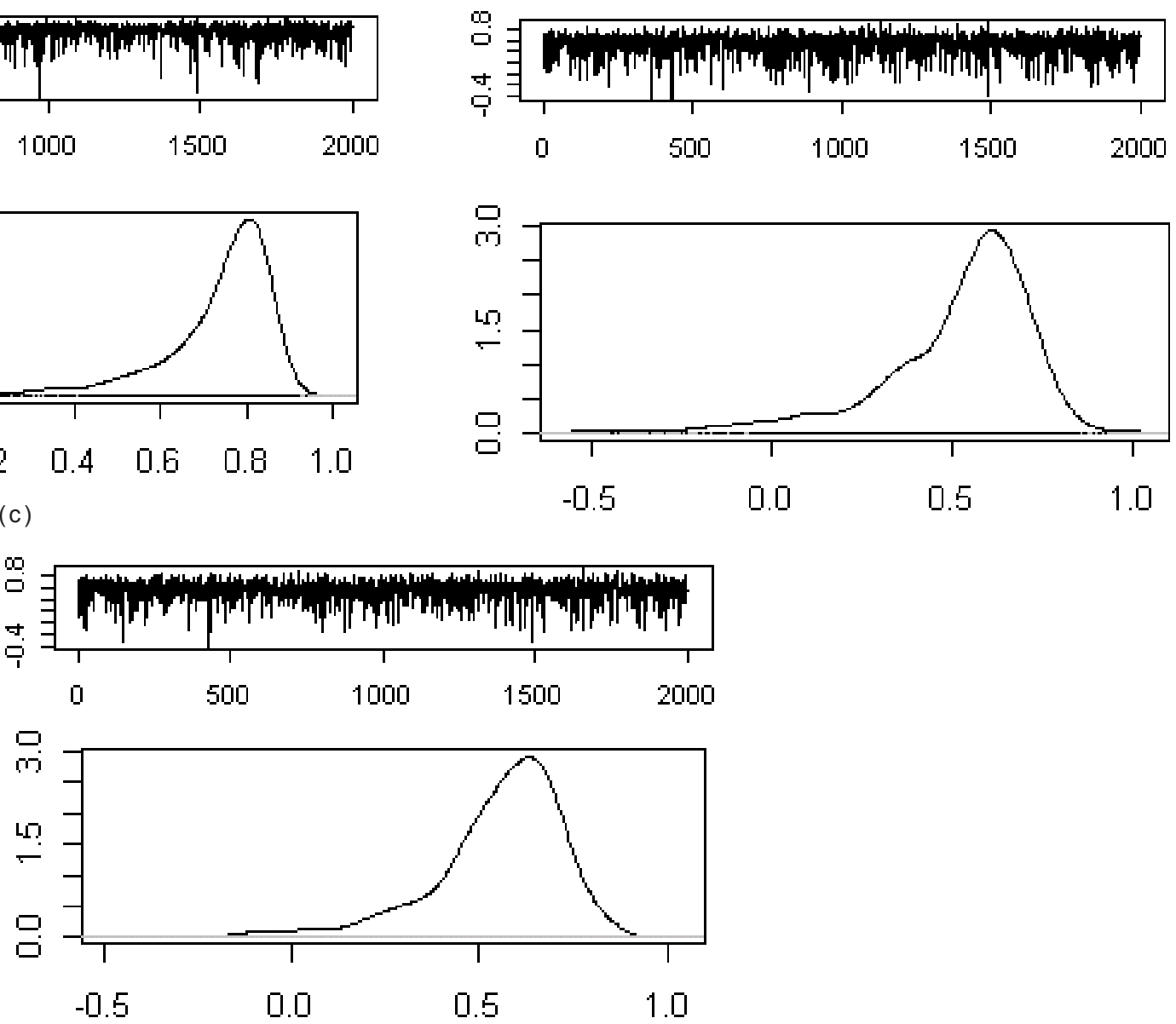

Figura 2 - Trajetória da cadeia e aproximação da distribuição a posteriori da correlação genética entre os pesos (a) aos 7 e 14 dias, (b) aos 7 e 28 dias e (c) aos 7 e 42 dias.

Figure 2 - Trajectory of the chain and approximation of posterior distribution of genetic correlations between: (a) weight at 7 and 14 days, (b) weight at 7 and 28 days and (c) weight at 7 and 42 days. 
(a)
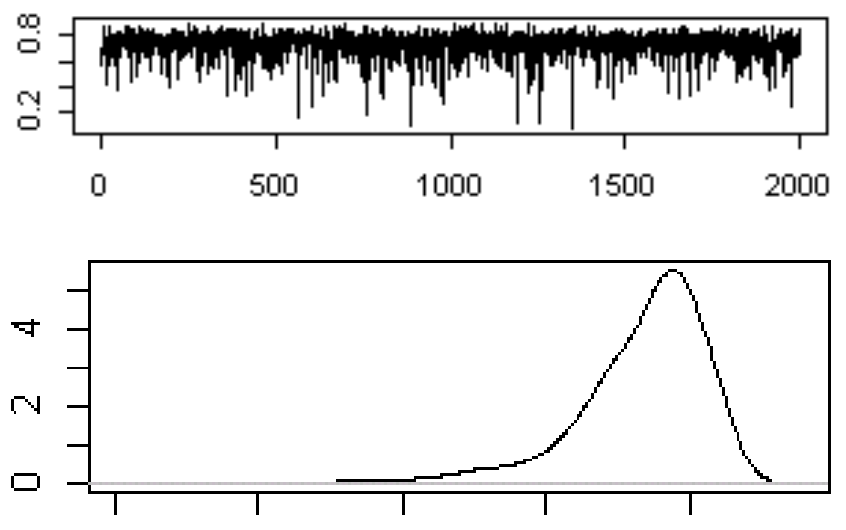

$\begin{array}{lllll}0.0 & 0.2 & 0.4 & 0.6 & 0.8\end{array}$

(b)
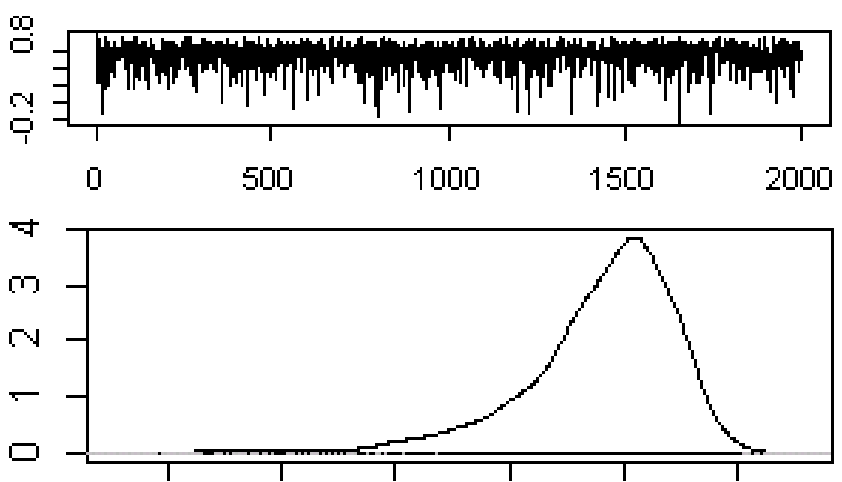

$\begin{array}{llllll}-0.2 & 0.0 & 0.2 & 0.4 & 0.6 & 0.8\end{array}$

(c)
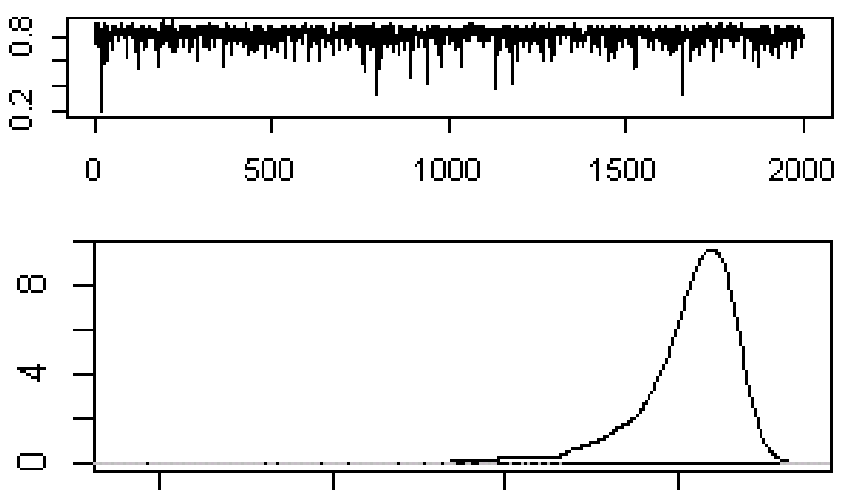

0.2

0.4

0.6

0.8

Figura 3 - Trajetória da cadeia e aproximação da densidade a posteriori da correlação genética entre os pesos: (a) aos 14 e 28 dias, (b) aos 14 e 42 dias e (c) aos 28 e 42 dias.

Figure 3 - Trajectory of the chain and approximation of posterior distribution of genetic correlations between: (a) weight at 14 and 28 days, (b) weight at 14 and 42 days and (c) weight at 28 and 42 days. as distribuições a posteriori (abaixo) das características analisadas são descritos nas Figuras 2 e 3.

As diferentes cadeias se aproximaram de um valor constante ao longo das iterações, indicando indícios de convergência (Figuras 2 e 3 ). Portanto, pode-se inferir que as estimativas das correlações genéticas são, verdadeiramente, as médias das distribuições a posteriori, pois a condição de estacionaridade foi atingida. Nota-se ainda nestes gráficos a presença de alguns valores baixos, que, provavelmente, causaram a assimetria à esquerda. As distribuições a posteriori das correlações genéticas entre os pesos aos 14 e 28 dias e aos 28 e 42 dias apresentaram menor dispersão, ou seja, as estimativas foram mais precisas.

\section{Conclusões}

As correlações genéticas obtidas entre os pesos nas diferentes idades analisadas indicam que a seleção para peso ao abate pode ser realizada em idades anteriores.

Os menores intervalos de credibilidade das estimativas de correlações genéticas foram entre os pesos aos 28 e 42 dias e aos 14 e 28 dias.

A metodologia Bayesiana utilizando-se amostrador de Gibbs permitiu a obtenção de estimativas pontuais e intervalares de correlações genéticas.

\section{Agradecimento}

Ao Conselho Nacional de Desenvolvimento Científico e Tecnológico (CNPq), pelo suporte financeiro, e à Universidade Federal do Paraná.

\section{Literatura Citada}

AGGREY, S.E.; CHENG, K.M. Animal model analysis of genetic (co)variances for growth traits in Japanese quail. Poultry Science, v.73, n.12, p.1822-1828, 1994

ALMEIDA, M.I.M.; OLIVEIRA, E.G.; RAMOS, P.R. et al. Efeito de linhagem e nível protéico sobre as características de carcaça de machos de codornas (Coturnix sp.). In: SIMPÓSIO NACIONAL DE MELHORAMENTO ANIMAL, 4., 2002, Campo Grande. Anais... Campo Grande: Embrapa Gado de Corte, 2002. p. 105-107.

ALMEIDA, M.I.M.; OLIVEIRA, E.G.; RAMOS, P.R. et al. Growth performance of meat male quails (Coturnix sp.) of two lines under two nutritional environments. Archives of Veterinary Science, v.7, n.2, p.103-108, 2002.

BRAH, G.S.; CHAUDHARY, M.L.; SANDHU, J.S. Genetic analysis of body weight in three lines of Japanese quails. Indian Journal of Poultry Science, v.32, n.3, p.242-248, 1997.

BURT, D.W. Applications of biotechnology in the poultry industry. World's Poultry Science, v.58, p.5-13, 2002.

FALCÃO, A.J.S.; MARTINS, E.N.; COSTA, C.N. et al. Aplicação de métodos REML e Bayesiano via amostrador de Gibbs na 
estimação de componentes de variância para produção de leite no estado do Paraná. In: SIMPÓSIO DA SOCIEDADE BRASILEIRA DE MELHORAMENTO ANIMAL, 5., 2004, Pirassununga. Anais... Pirassununga: Sociedade Brasileira de Melhoramento Animal, 2004. p.1.4.

KUMAR, A.; VERMA, S.B.; MOHAN, M. et al. Estimates of correlation among body weight at various weeks of age under different selection intensities in Japanese quails. Indian Veterinary Journal, v.79, n.1, p.41-43, 2002.

MARTINS, E.N. Novos conceitos aplicados à produção de codornas. In: SIMPÓSIO INTERNACIONAL DE COTURNICULTURA, 1., 2002, Lavras. Anais... Lavras: Universidade Federal de Lavras, 2002. p.109-112.

NATIONAL RESEARCH COUNCIL - NRC. Nutrient requirements of poultry. 9.ed. Washington, D.C.: National Academy Press, 1994. 156p.

OliVEIRA, E.G.; ALMEIDA, M.I.M.; MENDES, A.A. et al. Desempenho produtivo de codornas para corte de ambos os sexos alimentadas com dietas com quatro níveis protéicos. Archives of Veterinary Science, v.7, n.2, p.75-80, 2002.
OLIVEIRA, E.G.; ALMEIDA, M.I.M.; MENDES, A.A. et al. Avaliação sensorial de carne de codornas para corte, abatidas aos 35, 56 e 77 dias de idade. Veterinária e Zootecnia, v.12, n.1/2, p.61-68, 2005

SAATCI, M.; DEWI, I.A.; AKSOY, A.R. Application of REML procedure to estimate the genetic parameters of weekly liveweights in one-to-one sire and dam pedigree recorded Japanese quails. Journal of Animal Breeding and Genetics, v. 120 , n.1, p.23-28, 2003.

SEFTON, A.E.; SIEGEL, P.B. Inheritance of body weight in Japanese quail. Poultry Science, v.53, p.1597-1603, 1974.

Van TASSEL, C.P.; Van VLECK, L.D. Multiple-trait Gibbs sampler for animal models: flexible programs for bayesian and likelihood based (co)variance component inference. Journal of Animal Sciences, v.74, p.2586-2597, 1996. 